\title{
Optimal Distance between Mobile Buoy and Target for Moving Long Baseline Positioning System
}

\author{
Weisheng Yan ${ }^{1}$, Wei Chen ${ }^{1,2}$, Rongxin $\mathrm{Cui}^{1}$ and Huiping $\mathrm{Li}^{1}$ \\ ${ }^{1}$ (School of Marine Science and Technology, Northwestern Polytechnical University, \\ P.R. China) \\ ${ }^{2}$ (Department of Mechanical Engineering, University of Victoria, Canada) \\ (E-mail: chenw062569@163.com)
}

\begin{abstract}
This paper investigates the problem of how to design the distance between a mobile buoy and the target to derive maximum positioning accuracy with a Moving Long Baseline (MLBL). To that end, the positioning model and the error sources of MLBL are derived, respectively. It is assumed that the position measurement of the mobile buoy and the distance measurement between the mobile buoy and the target are corrupted by white Gaussian noises, and the variance of the distance measurement is distance-dependent. Using tools from estimation theory, the Positioning Accuracy Metric (PAM) is designed with the distance error and the position errors are considered. Based on the PAM, the optimal distance between the mobile buoy and target is deduced when the mobile buoys are in optimal geometry. Simulation examples illustrate the results.
\end{abstract}
KEY WORDS
1. MLBL.
2. Mobile buoy.
3. Optimal distance.
4. Positioning accuracy.

Submitted: 24 April 2014. Accepted: 18 January 2015. First published online: 23 February 2015.

1. INTRODUCTION. There has been increasing interest in the use of underwater vehicles to explore and exploit the ocean, such as mine-hunting, target or animal tracking, disaster response, and oceanographic surveys (Moradi et. al., 2012; Erol-Kantarci et. al., 2011; Waite, 2002). Precise positioning of underwater vehicles is important for the safety and effectiveness of all these autonomous missions. However, the underwater environment is complex and access-constrained, presenting unique challenges to accurate positioning compared with land and air environments. In the underwater environment, the popular Global Positioning System (GPS) is not feasible due to the strong attenuation that the electromagnetic field suffers in water (Sukkarieh et. al., 1999; Yun et. al., 1999). Therefore, acoustic-based positioning systems have been sought in the past, including systems such as Long Baseline (LBL), Short Baseline (SBL), and Ultra-Short Baseline (USBL) (Tan et. al., 2011; Olson et. al., 2006; Kinsey et. al., 2006). 
In LBL, a set of three or more baseline transponders are deployed on the sea floor, and the positions of the transponders must be determined in advance (Jakuba et. al., 2008). In SBL, three or more transponders are mounted on a surface vessel and connected to a central control station. In both systems, a trilateration algorithm is used to estimate the target position (Tan et. al., 2009). In USBL, a set of transponders assembled in a single device is installed on a support ship, and the target position is estimated by measuring the relative phases between the signals arriving at the transponders (Beaujean et. al., 2010; Philips, 2003). All of these systems have their merits and drawbacks. SBL and USBL require less infrastructure, but the positioning accuracy and the operational area are constrained. In comparison with SBL and USBL, LBL can achieve higher accuracy, but it has several shortcomings, e.g., requiring a long time for deployment and calibration, and a limited operating region.

Moving Long Baseline (MLBL) is a generalisation of LBL by replacing the pre-calibrated arrays of static transponders with self-calibrated and fully mobile transponders (Caiti et. al., 2005; Alcocer et. al., 2007). The absolute position of the target can be estimated by the Least Squares (LS) method based on the acoustic and GPS observations (Vaganay et. al., 2004; Kussat et. al., 2005). In this way, the MLBL overcomes the shortcomings of LBL. In the past few years, many algorithms for efficient target position estimation have been proposed in the literature, such as Extended Kalman Filtering (EKF) (Olson et. al., 2006; Batista et. al., 2014), Particle Filtering (PF) (Fox et. al., 2000), and Maximum Likelihood Estimation (MLE) (Howard et. al., 2002).

In MLBL, the absolute position of the target is calculated by using the position measurements of the mobile buoys and the distance measurements between the target and the mobile buoys. The positioning error may arise from three factors, i.e., the position error of the mobile buoy relative to the earth reference frame, the traveling time error and the deviation of actual sound speed from the assumed sound speed (Moradi et. al., 2012; Kinsey et. al., 2006; Isik and Akan, 2009; Liu et. al., 2010; Kaplan and Hegarty, 2005; Teymorian et. al., 2009). The distance between the mobile buoy and the target is calculated by the sound speed and the One-Way Travel Time (OWTT). Hence the positioning accuracy of the target is mainly affected by the position error of the mobile buoy and the distance error between the mobile buoy and the target. Considering that the positioning accuracy is only affected by the distance error, some researchers have found that the accuracy of the position estimates can be computed through the Cramer-Rao bound (CRB) and Fisher Information Matrix (FIM). Then the optimal geometry by minimizing the CRB or maximizing the FIM is provided (Van Trees, 2004; Savvides et. al., 2005; Alcocer et. al., 2006; Moreno-Salinas et. al., 2011; MartíNez and Bullo, 2006; Moreno-Salinas et. al., 2012; Purvis et. al., 2008; Oshman and Davidson, 1999; Xu and Choi, 2011). However, the position error of the mobile buoy is ignored. This work differs from the aforementioned approaches by considering that the positioning accuracy is affected by the distance error and the position error of the mobile buoy. According to the estimation theory, we propose a Positioning Accuracy Metric (PAM) to measure the positioning accuracy with the distance error and the position errors are also considered. We find that the positioning accuracy is related to the distance between the mobile buoy and the target. The optimal distance is determined by the depth of the target and the parameter of the distance error. 


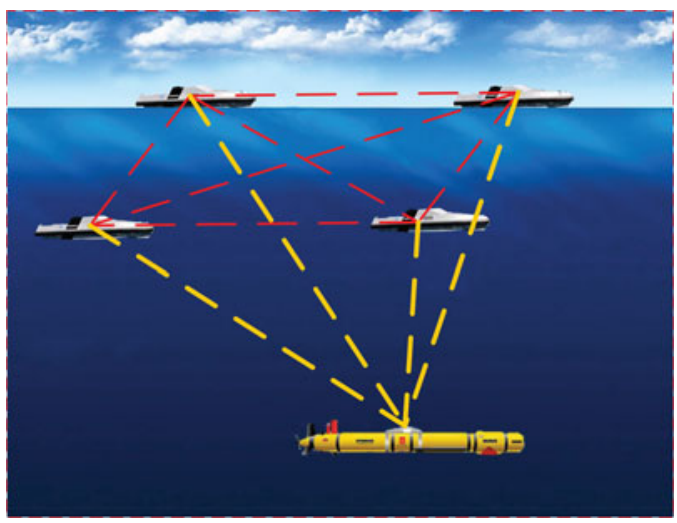

Figure 1. MLBL positioning system consists of four mobile buoys.

The remainder of the paper is organised as follows. Section 2 presents the mathematical model of MLBL. The PAM with the distance error and the position error considered is proposed in Section 3. Section 4 presents the optimal geometry and the optimal distance. Section 5 illustrates the simulation results. Finally, we conclude our work in Section 6.

2. MATHEMATICAL MODEL OF MLBL. As shown in Figure 1, the mobile buoys on the water surface are equipped with transponders, Differential GPS (DGPS), wireless communications, and the target is equipped with transponders. The position of the target is determined by the position measurements of the mobile buoys and the distance measurements between the target and each mobile buoy.

2.1. Kinematics model. As shown in Figure 2, consider an earth fixed reference frame $\{O\}:=\left\{x_{0}, y_{0}, z_{0}\right\}$ with $z=0$ on the water surface, and the $z$-axis pointing downward from the water surface. The coordinate of the target at stamp $k$ is $\left(x_{k}, y_{k}, z_{k}\right)$.

The kinematics model of the target is described as

$$
\left\{\begin{array}{l}
x_{k+1}=x_{k}+T_{s} v_{x k}=x_{k}+T_{s} v_{k} \cos \psi_{k} \cos \theta_{k}, \\
y_{k+1}=y_{k}+T_{s} v_{y k}=y_{k}+T_{s} v_{k} \sin \psi_{k} \cos \theta_{k}, \\
z_{k+1}=z_{k}+T_{s} v_{z k}=z_{k}+T_{s} v_{k} \sin \theta_{k},
\end{array}\right.
$$

where $T_{s}$ is the sampling period, $v_{k}$ is the velocity, $\psi_{k}$ is the yaw, $\theta_{k}$ is the pitch. Suppose there are $m$ mobile buoys on the water surface, their coordinates at stamp $k$ are $\left(x_{i, k}, y_{i, k}, z_{i, k}\right)$, with $z_{i, k}=0$. The kinematics model of each mobile buoy is described as

$$
\left\{\begin{array}{c}
x_{i, k+1}=x_{i, k}+T_{s} v_{i, k} \cos \psi_{i, k}, \\
y_{i, k+1}=y_{i, k}+T_{s} v_{i, k} \sin \psi_{i, k},
\end{array} \quad i=1, \ldots, m,\right.
$$

where $v_{i, k}$ is the velocity, $\psi_{i, k}$ is the yaw.

2.2. Positioning model. Assuming that the positions of the mobile buoys and the distances between the target and each mobile buoy are known, the estimated position 
the water surface

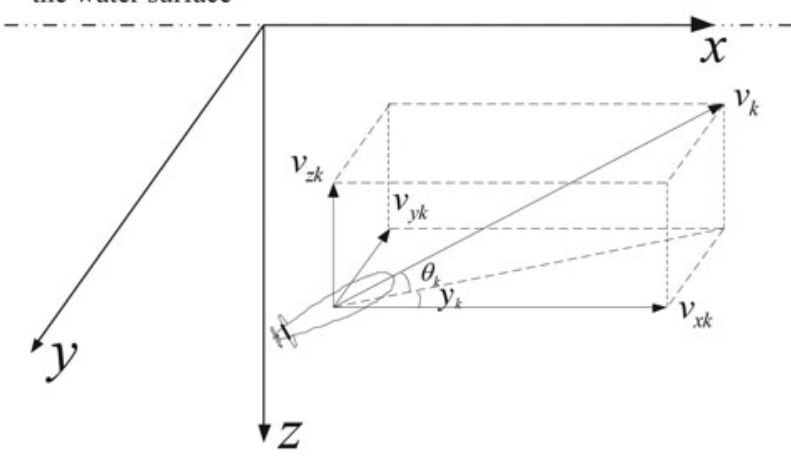

Figure 2. The kinematics model of the target.

of the target $\left(x_{k}, y_{k}, z_{k}\right)$ should satisfy

$$
\left(x_{k}-x_{i, k}\right)^{2}+\left(y_{k}-y_{i, k}\right)^{2}+\left(z_{k}-z_{i, k}\right)^{2}=r_{i, k}^{2}, i=1, \ldots, m .
$$

As mentioned before, the mobile buoys are on the water surface with $z_{i, k}=0$, then we have

$$
2\left(x_{i, k}-x_{j, k}\right) x_{k}+2\left(y_{i, k}-y_{j, k}\right) y_{k}=-r_{i, k}^{2}+r_{j, k}^{2}+x_{i, k}^{2}+y_{i, k}^{2}-x_{j, k}^{2}-y_{j, k}^{2}
$$

where $i \neq j, x_{k}$ and $y_{k}$ are unknown variables to be estimated. The positioning model can be expressed as

$$
\boldsymbol{A} \boldsymbol{X}=\boldsymbol{B},
$$

where

$$
\boldsymbol{A}=\left[\begin{array}{cc}
2\left(x_{1, k}-x_{2, k}\right) & 2\left(y_{1, k}-y_{2, k}\right) \\
2\left(x_{2, k}-x_{3, k}\right) & 2\left(y_{2, k}-y_{3, k}\right) \\
\vdots & \vdots \\
2\left(x_{m-1, k}-x_{m, k}\right) & 2\left(y_{m-1, k}-y_{m, k}\right) \\
2\left(x_{m, k}-x_{1, k}\right) & 2\left(y_{m, k}-y_{1, k}\right)
\end{array}\right], \boldsymbol{X}=\left[x_{k}, y_{k}\right]^{T},
$$

and

$$
\boldsymbol{B}=\left[\begin{array}{c}
-r_{1, k}^{2}+r_{2, k}^{2}+x_{1, k}^{2}+y_{1, k}^{2}-x_{2, k}^{2}-y_{2, k}^{2} \\
-r_{2, k}^{2}+r_{3, k}^{2}+x_{2, k}^{2}+y_{2, k}^{2}-x_{3, k}^{2}-y_{3, k}^{2} \\
\vdots \\
-r_{m-1, k}^{2}+r_{m, k}^{2}+x_{m-1, k}^{2}+y_{m-1, k}^{2}-x_{m, k}^{2}-y_{m, k}^{2} \\
-r_{m, k}^{2}+r_{1, k}^{2}+x_{m, k}^{2}+y_{m, k}^{2}-x_{1, k}^{2}-y_{1, k}^{2}
\end{array}\right] .
$$


3. POSITIONING ACCURACY METRIC (PAM). In this Section, we find that the positioning accuracy is related to the position error of the mobile buoy and the distance error between the mobile buoy and the target via the Partial Differential Equation (PDE). On this basis, we will design the PAM to measure the positioning accuracy.

3.1. Analysis of error sources. The differential form of Equation (4) is written as

$$
\begin{aligned}
& \left(x_{i, k}-x_{j, k}\right) \mathrm{d} x_{k}+\left(y_{i, k}-y_{j, k}\right) \mathrm{d} y_{k}+x_{k} \mathrm{~d} x_{i, k}-x_{k} \mathrm{~d} x_{j, k}+y_{k} \mathrm{~d} y_{i, k}-y_{k} \mathrm{~d} y_{j, k} \\
& =-r_{i, k} \mathrm{~d} r_{i, k}+r_{j, k} \mathrm{~d} r_{j, k}+x_{i, k} \mathrm{~d} x_{i, k}+y_{i, k} \mathrm{~d} y_{i, k}-x_{j, k} \mathrm{~d} x_{j, k}-y_{j, k} \mathrm{~d} y_{j, k} .
\end{aligned}
$$

In a compact form,

$$
\boldsymbol{C} \mathrm{d} \boldsymbol{X}=\mathrm{d} \boldsymbol{D}_{\text {station }}+\mathrm{d} \boldsymbol{D}_{\text {distance }},
$$

where

$$
\begin{gathered}
\boldsymbol{C}=\left[\begin{array}{cc}
\left(x_{1, k}-x_{2, k}\right) & \left(y_{1, k}-y_{2, k}\right) \\
\left(x_{2, k}-x_{3, k}\right) & \left(y_{2, k}-y_{3, k}\right) \\
\vdots & \vdots \\
\left(x_{m-1, k}-x_{m, k}\right) & \left(y_{m-1, k}-y_{m, k}\right) \\
\left(x_{m, k}-x_{1, k}\right) & \left(y_{m, k}-y_{1, k}\right)
\end{array}\right], \\
\mathrm{d} \boldsymbol{X}=\left[\mathrm{d} x_{k}, \mathrm{~d} y_{k}\right]^{T},
\end{gathered}
$$

$\mathrm{d} \boldsymbol{D}_{\text {station }}$

$$
=\left[\begin{array}{c}
\left(x_{1, k}-x_{k}\right) \mathrm{d} x_{1, k}+\left(y_{1, k}-y_{k}\right) \mathrm{d} y_{1, k}-\left(x_{2, k}-x_{k}\right) \mathrm{d} x_{2, k}-\left(y_{2, k}-y_{k}\right) \mathrm{d} y_{2, k} \\
\left(x_{2, k}-x_{k}\right) \mathrm{d} x_{2, k}+\left(y_{2, k}-y_{k}\right) \mathrm{d} y_{2, k}-\left(x_{3, k}-x_{k}\right) \mathrm{d} x_{3, k}-\left(y_{3, k}-y_{k}\right) \mathrm{d} y_{3, k} \\
\vdots \\
\left(x_{m-1, k}-x_{k}\right) \mathrm{d} x_{m-1, k}+\left(y_{m-1, k}-y_{k}\right) \mathrm{d} y_{m-1, k}-\left(x_{m, k}-x_{k}\right) \mathrm{d} x_{m, k}-\left(y_{m, k}-y_{k}\right) \mathrm{d} y_{m, k} \\
\left(x_{m, k}-x_{k}\right) \mathrm{d} x_{m, k}+\left(y_{m, k}-y_{k}\right) \mathrm{d} y_{m, k}-\left(x_{1, k}-x_{k}\right) \mathrm{d} x_{1, k}-\left(y_{1, k}-y_{k}\right) \mathrm{d} y_{1, k}
\end{array}\right],
$$

and

$$
\mathrm{d} \boldsymbol{D}_{\text {distance }}=\left[\begin{array}{c}
-r_{1, k} \mathrm{~d} r_{1, k}+r_{2, k} \mathrm{~d} r_{2, k} \\
-r_{2, k} \mathrm{~d} r_{2, k}+r_{3, k} \mathrm{~d} r_{3, k} \\
\vdots \\
-r_{m-1, k} \mathrm{~d} r_{m-1, k}+r_{m, k} \mathrm{~d} r_{m, k} \\
-r_{m, k} \mathrm{~d} r_{m, k}+r_{1, k} \mathrm{~d} r_{1, k}
\end{array}\right] .
$$

According to the LS algorithm, we have

$$
\mathrm{d} \boldsymbol{X}=\left(\boldsymbol{C}^{T} \boldsymbol{C}\right)^{-1} \boldsymbol{C}^{T}\left(\mathrm{~d} \boldsymbol{D}_{\text {station }}+\mathrm{d} \boldsymbol{D}_{\text {distance }}\right) .
$$

From Equation (14), it can be seen that the positioning error of the target $\mathrm{d} \boldsymbol{X}$ is related to the distance error $\mathrm{d} \boldsymbol{D}_{\text {distance }}$ and the position error of the mobile buoy $\mathrm{d} \boldsymbol{D}_{\text {station }}$.

3.2. Derivation of $P A M$. In the water, the distance error is caused by variable sound speed, physical propagation barriers, ambient noise, and degrading signal-to- 
noise ratio as the distance between the two objects increases. It is commonly assumed that the distance measurement can be captured by white Gaussian noise, the variance of which is distance-dependent (Moreno-Salinas et. al., 2011; Jourdan and Roy, 2008). The distance error at stamp $k$ is established,

$$
\boldsymbol{\varepsilon}_{r, k}=\left(\boldsymbol{I}+\eta \boldsymbol{r}_{k}\right) \boldsymbol{\varepsilon}_{r},
$$

where $\boldsymbol{\varepsilon}_{r, k}=\operatorname{diag}\left\{\varepsilon_{1, k}, \boldsymbol{\varepsilon}_{2, k}, \ldots, \boldsymbol{\varepsilon}_{m, k}\right\}$ and $\boldsymbol{r}_{k}=\operatorname{diag}\left\{r_{1, k}, r_{2, k}, \ldots, r_{m, k}\right\} . \varepsilon_{i, k}$ is a Gaussian stochastic process with $\varepsilon_{i, k} \sim N\left(0, \sigma_{r_{i, k}}^{2}\right), \varepsilon_{r}$ is a Gaussian stochastic process with $\boldsymbol{\varepsilon}_{r} \sim N$ $\left(0, \sigma_{r}^{2} I\right)$, and $\eta$ is the parameter for the distance-dependent error component. The distance error should satisfy

$$
\sigma_{r_{i, k}}^{2}=\left(1+\eta r_{i, k}\right)^{2} \sigma_{r}^{2}
$$

The mobile buoys are on the water surface, and they are positioned by GPS. The position error of the mobile buoy can be captured by Gaussian zero mean additive noises with constant covariance. The position error of the mobile buoy $\left(x_{i, k}, y_{i, k}\right)$ is described as $\left(\varepsilon_{x_{i, k}}, \varepsilon_{y_{i, k}}\right)$, and $\left(\varepsilon_{x_{i, k}}, \varepsilon_{y_{i, k}}\right)$ is a zero mean Gaussian process with $\varepsilon_{x_{i, k}} \sim N\left(0, \sigma_{x_{i, k}}^{2}\right)$, $\varepsilon_{y_{i, k}} \sim N\left(0, \sigma_{y_{i, k}}^{2}\right)$. It is commonly assumed that

$$
\sigma_{s}^{2}=\sigma_{x_{i, k}}^{2}=\sigma_{y_{i, k}}^{2}=\sigma_{x_{j, k}}^{2}=\sigma_{y_{j, k}}^{2}
$$

The distance error and the position error are uncorrelated, then the covariance matrix of $\mathrm{d} \boldsymbol{X}$ is obtained as

$$
\boldsymbol{Q}=E\left[\mathrm{~d} \boldsymbol{X} \mathrm{d} \boldsymbol{X}^{T}\right]=\boldsymbol{G F} \boldsymbol{G}^{T}
$$

where

$$
\begin{gathered}
\boldsymbol{G}=\left(\boldsymbol{C}^{T} \boldsymbol{C}\right)^{-1} \boldsymbol{C}^{T} \\
\boldsymbol{F}=E\left[\mathrm{~d} \boldsymbol{D}_{\text {station }} \mathrm{d} \boldsymbol{D}_{\text {station }}^{T}\right]+E\left[\mathrm{~d} \boldsymbol{D}_{\text {distance }} \mathrm{d} \boldsymbol{D}_{\text {distance }}^{T}\right] .
\end{gathered}
$$

The covariance matrix of $\mathrm{d} \boldsymbol{D}_{\text {station }}$ is written as

$$
E\left[\mathrm{~d} \boldsymbol{D}_{\text {station }} \mathrm{d} \boldsymbol{D}_{\text {station }}^{T}\right]=\left[\begin{array}{ccccc}
\sigma_{X_{1}}^{2}+\sigma_{X_{2}}^{2} & * & * & * & * \\
* & \sigma_{X_{2}}^{2}+\sigma_{X_{3}}^{2} & * & * & * \\
* & * & \ddots & * & * \\
* & * & * & \sigma_{X_{m-1}}^{2}+\sigma_{X_{m}}^{2} & * \\
* & * & * & * & \sigma_{X_{m}}^{2}+\sigma_{X_{1}}^{2}
\end{array}\right]
$$

where

$$
\sigma_{X_{i}}^{2}=\left(x_{i, k}-x_{k}\right)^{2} \sigma_{x_{i, k}}^{2}+\left(y_{i, k}-y_{k}\right)^{2} \sigma_{y_{i, k}}^{2} .
$$

Define $r_{i, k}^{2}=z_{k}^{2}+R_{i, k}^{2}$, then we have

$$
\sigma_{X_{i}}^{2}=R_{i, k}^{2} \sigma_{s}^{2}
$$


It follows that

$$
\begin{aligned}
& E\left[\mathrm{~d} \boldsymbol{D}_{\text {station }} \mathrm{d} \boldsymbol{D}_{\text {station }}^{T}\right] \\
& =\left[\begin{array}{ccccc}
\left(R_{1, k}^{2}+R_{2, k}^{2}\right) \sigma_{s}^{2} & * & * & * & * \\
* & \left(R_{2, k}^{2}+R_{3, k}^{2}\right) \sigma_{s}^{2} & * & * & * \\
* & * & \ddots & * & * \\
* & * & * & \left(R_{m-1, k}^{2}+R_{m, k}^{2}\right) \sigma_{s}^{2} & * \\
* & * & * & * & \left(R_{m, k}^{2}+R_{1, k}^{2}\right) \sigma_{s}^{2}
\end{array}\right]
\end{aligned}
$$

The covariance matrix of $\mathrm{d} \boldsymbol{D}_{\text {distance }}$ is written as

$$
\begin{aligned}
& E\left[\mathrm{~d} \boldsymbol{D}_{\text {distance }} \mathrm{d} \boldsymbol{D}_{\text {distance }}^{T}\right] \\
& =\left[\begin{array}{ccccc}
r_{1, k}^{2} \sigma_{r_{1, k}}^{2}+r_{2, k}^{2} \sigma_{r_{2, k}}^{2} & * & * & * & * \\
* & r_{2, k}^{2} \sigma_{r_{2, k}}^{2}+r_{3, k}^{2} \sigma_{r_{3, k}}^{2} & * & * & * \\
* & * & \ddots & * & * \\
* & * & * & r_{m-1, k}^{2} \sigma_{r_{m-1, k}^{2}}^{2}+r_{m, k}^{2} \sigma_{r_{m, k}}^{2} & * \\
* & * & * & * & r_{m, k}^{2} \sigma_{r_{m, k}}^{2}+r_{1, k}^{2} \sigma_{r_{1, k}}^{2}
\end{array}\right]
\end{aligned}
$$

The optimal positioning accuracy can be determined by minimizing the trace of the covariance matrix with respect to the exteroceptive measurements (Lütkepohl, 1996). As a result, we have

$$
\operatorname{tr}(\boldsymbol{Q})=\operatorname{tr}\left(\boldsymbol{G F} \boldsymbol{G}^{T}\right)=\operatorname{tr}\left(\boldsymbol{F} \boldsymbol{G}^{T} \boldsymbol{G}\right) \leq \operatorname{tr}(\boldsymbol{F}) \operatorname{tr}\left(\boldsymbol{G}^{T} \boldsymbol{G}\right),
$$

where

$$
\begin{gathered}
\operatorname{tr}(\boldsymbol{F})=2 \sigma_{s}^{2} \sum_{i=1}^{m} R_{i, k}^{2}+2 \sigma_{r}^{2} \sum_{i=1}^{m}\left(1+\eta r_{i, k}\right)^{2} r_{i, k}^{2} \\
=2 \sigma_{s}^{2} \sum_{i=1}^{m}\left(r_{i, k}^{2}-z_{k}^{2}\right)+2 \sigma_{r}^{2} \sum_{i=1}^{m}\left(1+\eta r_{i, k}\right)^{2} r_{i, k}^{2}, \\
\operatorname{tr}\left(\boldsymbol{G}^{T} \boldsymbol{G}\right)=\operatorname{tr}\left(\left(\boldsymbol{C}^{T} \boldsymbol{C}\right)^{-1}\right) .
\end{gathered}
$$

According to Equation (10), we have

$$
\boldsymbol{C}^{T} \boldsymbol{C}=\left[\begin{array}{cc}
\sum_{i=1}^{m} a_{i, k}^{2} & \sum_{i=1}^{m} a_{i, k} b_{i, k} \\
\sum_{i=1}^{m} a_{i, k} b_{i, k} & \sum_{i=1}^{m} b_{i, k}^{2}
\end{array}\right],
$$


and

$$
\left\{\begin{array}{l}
a_{i, k}=x_{i, k}-x_{i+1, k} \\
a_{m, k}=x_{m, k}-x_{1, k}, \quad i=1, \ldots, m-1 . \\
b_{i, k}=y_{i, k}-y_{i+1, k}, \\
b_{m, k}=y_{m, k}-y_{1, k}
\end{array}\right.
$$

It follows that

$$
\operatorname{tr}\left(\left(\boldsymbol{C}^{T} \boldsymbol{C}\right)^{-1}\right)=\frac{\sum_{i=1}^{m} a_{i, k}^{2}+\sum_{i=1}^{m} b_{i, k}^{2}}{\left(\sum_{i=1}^{m} a_{i, k}^{2}\right)\left(\sum_{i=1}^{m} b_{i, k}^{2}\right)-\left(\sum_{i=1}^{m} a_{i, k} b_{i, k}\right)^{2}} .
$$

Then the PAM function $J$ can be described as

$$
\begin{aligned}
& J=\operatorname{tr}(\boldsymbol{F}) \operatorname{tr}\left(\boldsymbol{G}^{T} \boldsymbol{G}\right)=\operatorname{tr}(\boldsymbol{F}) \operatorname{tr}\left(\left(\boldsymbol{C}^{T} \boldsymbol{C}\right)^{-1}\right) \\
& =\left[2 \sigma_{s}^{2} \sum_{i=1}^{m}\left(r_{i, k}^{2}-z_{k}^{2}\right)+2 \sigma_{r}^{2} \sum_{i=1}^{m}\left(1+\eta r_{i, k}\right)^{2} r_{i, k}^{2}\right] \cdot \frac{\sum_{i=1}^{m} a_{i, k}^{2}+\sum_{i=1}^{m} b_{i, k}^{2}}{\left(\sum_{i=1}^{m} a_{i, k}^{2}\right)\left(\sum_{i=1}^{m} b_{i, k}^{2}\right)-\left(\sum_{i=1}^{m} a_{i, k} b_{i, k}\right)^{2}} .
\end{aligned}
$$

4. OPTIMAL DISTANCE. In this Section, we will derive the optimal distance by minimizing the $J$ that was established in Section 3.2. We find that the positioning accuracy is related to the geometry of the mobile buoys and the distances between the target and each mobile buoy.

4.1. Derivation of optimal geometry. The PAM $J$ is composed of two parts: $\operatorname{tr}(F)$ and $\operatorname{tr}\left(\left(C^{T} C\right)^{-1}\right)$. From Equation (27), it can be seen that $\operatorname{tr}(F)$ is irrelevant to the geometry of the mobile buoys. So our work is to minimize $\operatorname{tr}\left(\left(C^{T} C\right)^{-1}\right)$. By defining $p_{1}=\sum_{i=1}^{m} a_{i, k}^{2}, p_{2}=\sum_{i=1}^{m} b_{i, k}^{2}, p_{3}=\sum_{i=1}^{m} a_{i, k} b_{i, k}$ and $P\left(p_{1}, p_{2}, p_{3}\right)=\operatorname{tr}\left(\left(C^{T} C\right)^{-1}\right)$, Equation (31) can be written as

$$
P\left(p_{1}, p_{2}, p_{3}\right)=\frac{p_{1}+p_{2}}{p_{1} p_{2}-p_{3}^{2}} .
$$

According to the Lagrange multiplier method (Bertsekas, 1982), we know that the $P$ $\left(p_{1}, p_{2}, p_{3}\right)$ reaches the minimum value when $p_{1}, p_{2}$ and $p_{3}$ satisfy

$$
\left\{\begin{array}{l}
\frac{\partial P\left(p_{1}, p_{2}, p_{3}\right)}{\partial p_{1}}=0 \\
\frac{\partial P\left(p_{1}, p_{2}, p_{3}\right)}{\partial p_{2}}=0 \\
\frac{\partial P\left(p_{1}, p_{2}, p_{3}\right)}{\partial p_{3}}=0
\end{array}\right.
$$




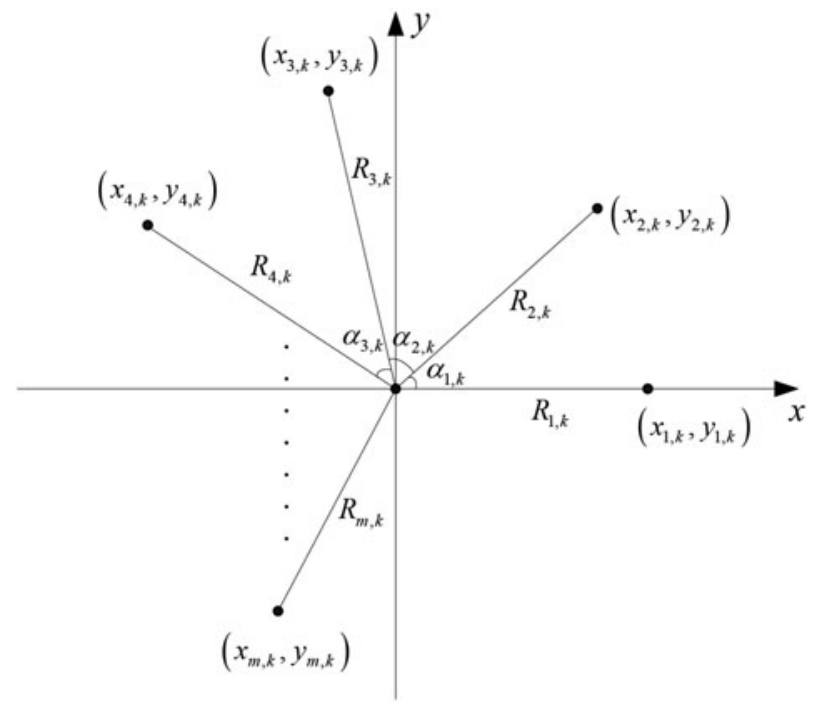

Figure 3. Positions of the target and the mobile buoys.

Thus, we have

$$
\left\{\begin{array}{c}
p_{1}=p_{2}, \\
p_{3}=0 .
\end{array}\right.
$$

This means that the $\operatorname{tr}\left(\left(C^{T} C\right)^{-1}\right)$ reaches the minimum value when

$$
\left\{\begin{array}{c}
\sum_{i=1}^{m} a_{i, k}^{2}=\sum_{i=1}^{m} b_{i, k}^{2}, \\
\sum_{i=1}^{m} a_{i, k} b_{i, k}=0 .
\end{array}\right.
$$

From Equation (30), it can be seen that $a_{i, k}$ and $b_{i, k}$ are determined by the positions of the mobile buoys, and Equation (36) can be reached by adjusting the positions of mobile buoys. Here, we use Figure 3 to illustrate how to reach Equation (36). As shown in Figure 3, a 2-dimensional (2D) Cartesian is established. $O$ is the projection of the target to the horizontal plane, $\left(x_{1, k}, y_{1, k}\right)$ is in the axis of abscissas, $\alpha_{i, k}$ is the angular distance between

$\left(x_{i, k}, y_{i, k}\right)$ and $\left(x_{i+1, k}, y_{i+1, k}\right)$ measured from $\left(x_{k}, y_{k}\right)$. Define $\alpha_{0, k}=0$, then we have

$$
\left\{\begin{array}{l}
a_{i, k}=R_{i, k} \cos \left(\sum_{n=0}^{i-1} \alpha_{n, k}\right)-R_{i+1, k} \cos \left(\sum_{n=0}^{i} \alpha_{n, k}\right), \\
a_{m, k}=R_{m, k} \cos \left(\sum_{n=0}^{m-1} \alpha_{n, k}\right)-R_{1, k} \cos \left(\sum_{n=0}^{m} \alpha_{n, k}\right), \\
b_{i, k}=R_{i, k} \sin \left(\sum_{n=0}^{i-1} \alpha_{n, k}\right)-R_{i+1, k} \sin \left(\sum_{n=0}^{i} \alpha_{n, k}\right), \\
b_{m, k}=R_{m, k} \sin \left(\sum_{n=0}^{m-1} \alpha_{n, k}\right)-R_{1, k} \sin \left(\sum_{n=0}^{m} \alpha_{n, k}\right),
\end{array}\right.
$$




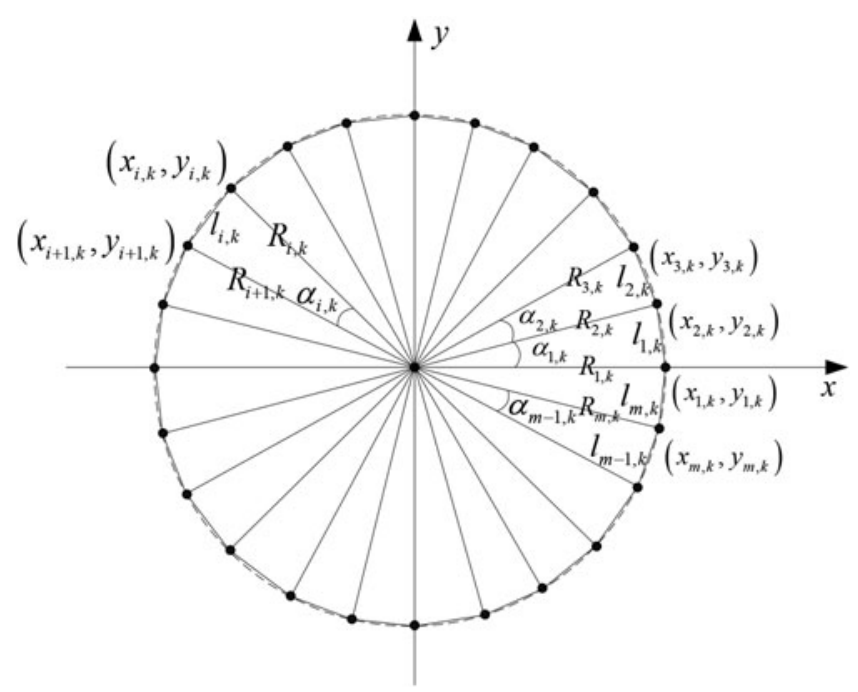

Figure 4. Positions of the target and the mobile buoys in the optimal geometry.

Equation (36) is satisfied when

$$
\left\{\begin{array}{c}
\alpha_{i, k}=\frac{2 \pi}{m}, \\
R_{1, k}=R_{2, k}=\ldots=R_{m, k} .
\end{array}\right.
$$

From this, we can see that the optimal geometry is that the mobile buoys are on the vertices of a regular polygon centred at the target.

4.2. PAM in optimal geometry. In this sub-section, we will calculate the PAM when the optimal geometry has been reached. Here, we use Figure 4 to illustrate how to calculate the PAM. As shown in Figure 4, $m$ mobile buoys are on the vertices of a regular polygon, $R_{i, k}$ is the circumradius of the regular $m$-gons, and $l_{i, k}$ is the side length (Williams, 1996). By defining $R=R_{i, k}$, we have

$$
\begin{gathered}
l_{i, k}=2 R \sin \frac{\pi}{m} \\
\left\{\begin{array}{l}
l_{i, k}^{2}=\left(x_{i, k}-x_{i+1, k}\right)^{2}+\left(y_{i, k}-y_{i+1, k}\right)^{2}=a_{i, k}^{2}+b_{i, k}^{2}, \quad i=1,2, \ldots, m-1, \\
l_{m, k}^{2}=\left(x_{m, k}-x_{1, k}\right)^{2}+\left(y_{m, k}-y_{1, k}\right)^{2}=a_{m, k}^{2}+b_{m, k}^{2},
\end{array}\right.
\end{gathered}
$$

and

$$
\sum_{i=1}^{m} l_{i, k}^{2}=\sum_{i=1}^{m} a_{i, k}^{2}+\sum_{i=1}^{m} b_{i, k}^{2} .
$$


Combining Equations (36)-(41) with Equation (31), it follows that

$$
\min _{\left(x_{i, k}, y_{i, k}\right) \in \mathbb{R}^{2}} \operatorname{tr}\left(\left(\boldsymbol{C}^{T} \boldsymbol{C}\right)^{-1}\right)=\frac{4}{\sum_{i=1}^{m} l_{i, k}^{2}}=\frac{1}{m\left(R \sin \frac{\pi}{m}\right)^{2}} .
$$

Thus, the PAM function $J$ in optimal geometry can be described as

$$
J=\left[2 \sigma_{s}^{2} \sum_{i=1}^{m}\left(r_{i, k}^{2}-z_{k}^{2}\right)+2 \sigma_{r}^{2} \sum_{i=1}^{m}\left(1+\eta r_{i, k}\right)^{2} r_{i, k}^{2}\right] \cdot \frac{1}{m\left(R \sin \frac{\pi}{m}\right)^{2}}
$$

4.3. Derivation of optimal distance. In this sub-section, we will study how to derive the optimal distance between the target and each mobile buoy. According to the Pythagorean theorem $r_{i, k}^{2}=z_{k}^{2}+R_{i, k}^{2}$, we have

$$
r_{1, k}=r_{2, k}=\ldots=r_{m, k} .
$$

By defining $r=r_{i, k}, \sigma_{s}^{2}=\mu \sigma_{r}^{2}$ and $z_{k}=h$, it follows that

$$
\begin{aligned}
& \min _{\left(x_{i, k}, y_{i, k}\right) \in \mathbb{R}^{2}} J=\min _{\left(x_{i, k}, y_{i, k}\right) \in \mathbb{R}^{2}} \operatorname{tr}(\boldsymbol{F}) \operatorname{tr}\left(\boldsymbol{G}^{T} \boldsymbol{G}\right)=\operatorname{tr}(\boldsymbol{F}) \operatorname{tr}\left(\left(\boldsymbol{C}^{T} \boldsymbol{C}\right)^{-1}\right) \\
= & \min _{\left(x_{i, k}, y_{i, k}\right) \in \mathbb{R}^{2}}\left[2 \sigma_{s}^{2} \sum_{i=1}^{m}\left(r_{i, k}^{2}-z_{k}^{2}\right)+2 \sigma_{r}^{2} \sum_{i=1}^{m}\left(1+\eta r_{i, k}\right)^{2} r_{i, k}^{2}\right] \cdot \frac{1}{m\left(R \sin \frac{\pi}{m}\right)^{2}} \\
= & \min _{\left(x_{i, k}, y_{i, k}\right) \in \mathbb{R}^{2}} 2 m \sigma_{r}^{2}\left[\mu\left(r^{2}-h^{2}\right)+(1+\eta r)^{2} r^{2}\right] \cdot \frac{1}{m\left(R \sin \frac{\pi}{m}\right)^{2}} \\
= & \min _{\left(x_{i, k}, y_{i, k}\right) \in \mathbb{R}^{2}} \frac{2 \sigma_{r}^{2}}{\left(\sin \frac{\pi}{m}\right)^{2}} \cdot\left[\mu+\frac{(1+\eta r)^{2} r^{2}}{r^{2}-h^{2}}\right] \\
= & d . \min _{\left(x_{i, k}, y_{i, k}\right) \in \mathbb{R}^{2}} g(\mu, \eta, h, r) .
\end{aligned}
$$

where

$$
\begin{aligned}
d & =\frac{2 \sigma_{r}^{2}}{\left(\sin \frac{\pi}{m}\right)^{2}}, \\
g(\mu, \eta, h, r) & =\left[\mu+\frac{(1+\eta r)^{2} r^{2}}{r^{2}-h^{2}}\right] .
\end{aligned}
$$

Note that $d$ is a constant, therefore the minimum value of $J$ is determined by $g(\mu, \eta, h, r)$. The optimal distance $r$ between target and each mobile buoy can be described when the parameter $\mu, \eta$ and the depth of the target $h$ are known. The differential equation of $g$ $(\mu, \eta, h, r)$ is written as

$$
\frac{\partial g}{\partial r}=\frac{2 r(1+\eta r)\left(\eta r^{3}-2 \eta h^{2} r-h^{2}\right)}{\left(r^{2}-h^{2}\right)^{2}}=l \cdot\left(\eta r^{3}-2 \eta h^{2} r-h^{2}\right)
$$


where

$$
l=\frac{2 r(1+\eta r)}{\left(r^{2}-h^{2}\right)^{2}}>0 .
$$

The minimum value of $J$ is reached when $\partial g / \partial r=0$. Assuming $\partial g / \partial r=0$, the optimal distance can be calculated by the Cardan method (Nickalls, 1993), then we have

$$
r=\left[\left(\frac{h^{4}}{4 \eta^{2}}-\frac{8 h^{6}}{27}\right)^{\frac{1}{2}}+\frac{h^{2}}{2 \eta}\right]^{\frac{1}{3}}+\frac{2 h^{2}}{3\left[\left(\frac{h^{4}}{4 \eta^{2}}-\frac{8 h^{6}}{27}\right)^{\frac{1}{2}}+\frac{h^{2}}{2 \eta}\right]^{\frac{1}{3}}}
$$

From Equation (45) and Equation (50), it can be seen that the positioning accuracy is determined by the geometry of mobile buoys and the distance between the mobile buoy and the target. The optimal geometry is that the mobile buoys are on the vertices of a regular polygon centred at the target, and the optimal distance is related to the parameter $\eta$ and the depth of the target $h$.

5. SIMULATIONS. This section describes two simulations to illustrate the optimal distance for the target positioning. In this simulation, Unmanned Surface Vehicles (USVs) equipped with transponders, GPS and wireless communications are employed as the mobile buoys, and an Autonomous Underwater Vehicle (AUV) equipped with a transponder is employed as the target. The positioning system consists of four USVs and an AUV. We assume that the distance error $\varepsilon_{i, k}$ between USV and AUV is around $1 \mathrm{~m}$ when this distance is small, and the distance error is about $0.1 \%$ of the distance when the distance is large. Then we can choose the parameters as $\sigma_{r}$ $=\varepsilon_{r}^{2}=\varepsilon_{i, r}^{2}=1$ and $\eta=\varepsilon_{i, r} / \varepsilon_{r} r_{i}=0.001 r_{i} / \varepsilon_{r} r_{i}=0.001 \mathrm{~m}^{-1}$. The parameter $\sigma_{r}$ increases along with the distance error when the distance is small, and the parameter $\eta$ also increases as distance error increases when the distance is large. The position errors of the USVs are assumed to be $\sigma_{s}=1$. The first example shows the evaluation of the PAM. The second example presents the evaluation through Monte Carlo method.

\subsection{Evaluation of PAM}

a) Optimal geometry: Figures 5 and 6 show the evaluations of the PAM.

In Figure 5, the AUV is static and the USVs are moving. The parameters of the AUV and the USVs are shown in Table 1, and the USVs are on the vertices of the square when $t=200 \mathrm{~s}$. As shown in Figure 5(a), the initial positions and the positions at $t=$ $200 \mathrm{~s}$ of the USVs are denoted by ' $\boldsymbol{\Lambda}$ ' and ' $\boldsymbol{\square}$ ', respectively. Figure 5(b) shows that the minimum value of the PAM is reached when $t=200 \mathrm{~s}$, with $J=12.477$.

In Figure 6, the static USVs are in the positions of $(400 \mathrm{~m}, 0 \mathrm{~m}, 0 \mathrm{~m}),(0 \mathrm{~m}, 400 \mathrm{~m}$, $0 \mathrm{~m}),(-400 \mathrm{~m}, 0 \mathrm{~m}, 0 \mathrm{~m}),(0 \mathrm{~m},-400 \mathrm{~m}, 0 \mathrm{~m})$, and the positions of the USVs are denoted by ' $*$ '. The AUV moves in a bounded region $(x, y, z) \mid-800 \mathrm{~m} \leq x \leq 800 \mathrm{~m}$, $-800 \mathrm{~m} \leq y \leq 800 \mathrm{~m}, z=100 \mathrm{~m}$. The results show that the minimum value of the PAM is reached when $(x, y)=(0 \mathrm{~m}, 0 \mathrm{~m})$, with $J=12.477$.

These results verify that the minimum value of the PAM is reached when the USVs are on the vertices of the square centred at the AUV. 
(a)

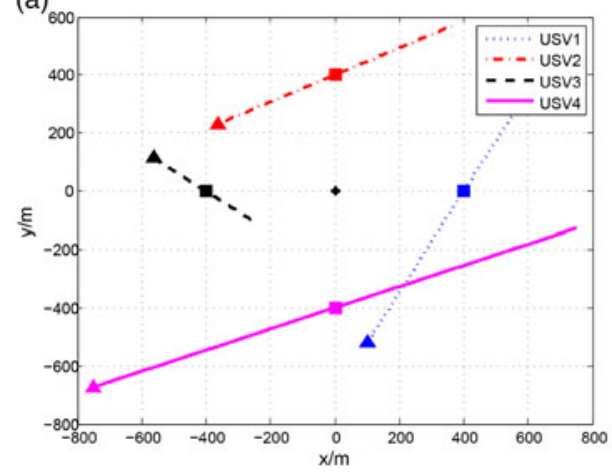

(b)

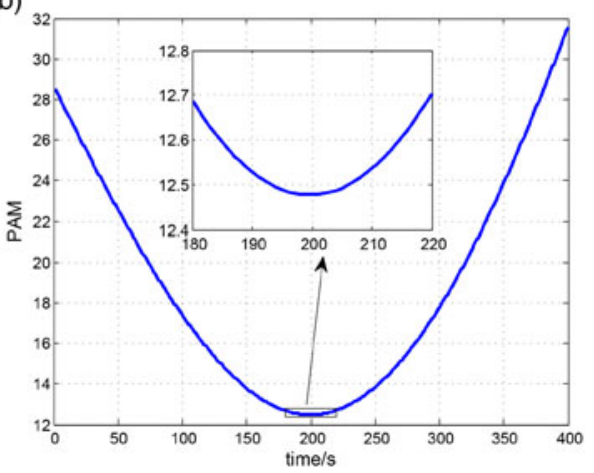

Figure 5. The evaluation of the PAM when USVs are moving: (a) the trajectories of the USVs, (b) the value of the PAM.

(a)

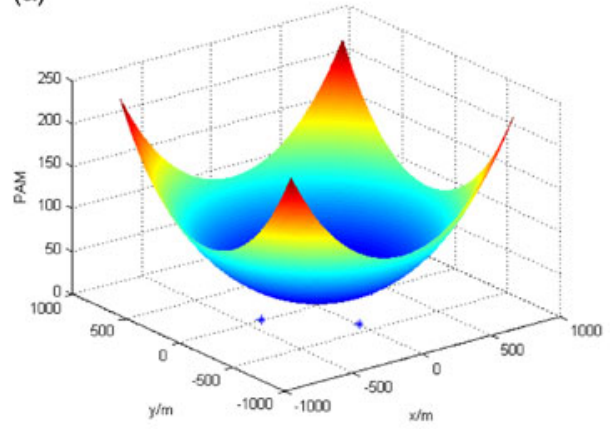

(b)

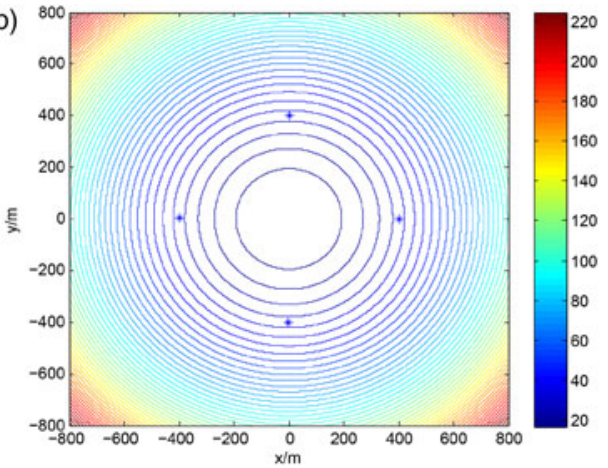

Figure 6. The evaluation of the PAM when AUV is moving: (a) 3-dimensional mesh graph, (b) contour graph

b) Optimal distance: In this example, the optimal geometry has been reached. We assume that the AUV is at a constant depth of $100 \mathrm{~m}$, and the distances between the AUV and each USV change. As shown in Figure 7, the PAM reaches the minimum value with $J=11.439$ when the distance is $r=246.221 \mathrm{~m}$. According to Equation (50), the optimal distance is $r=246.205 \mathrm{~m}$, and the slight difference is caused by the calculation. The value of the PAM is $J=12.477$ when the distance is $r=\sqrt{400^{2}+100^{2}}=412.311 \mathrm{~m}$, and it coincides with the result in part (a).

5.2. Evaluation through Monte Carlo method. Since the position errors of the USVs and AUV are random in nature, we compare the positioning errors by using Monte Carlo method. In this part, the positioning errors are simulated for 100 Monte Carlo trials. Firstly, we calculate the positioning errors $e_{q, k}=$ $\sqrt{\left(x_{k}^{q}-\tilde{x}_{k}^{q}\right)^{2}+\left(y_{k}^{q}-\tilde{y}_{k}^{q}\right)^{2}}$ of the AUV in 100 experiments at stamp $k$, where $\left(x_{k}^{q}, y_{k}^{\mathrm{q}}\right)$ and $\left(\tilde{x}_{k}^{q}, \tilde{y}_{k}^{q}\right)$ are the real and estimated positions of the AUV. Then the mean 
Table 1. The parameters of the USVs and the AUV.

\begin{tabular}{lcccc}
\hline Vehicle & Initial Position $(\mathrm{m})$ & Velocity $(\mathrm{m} / \mathrm{s})$ & Yaw $\left({ }^{\circ}\right)$ & Position at $t=200 \mathrm{~s}(\mathrm{~m})$ \\
\hline AUV & $(0,0,100)$ & 0 & 0 & $(0,0,100)$ \\
USV1 & $(100,-519 \cdot 62,0)$ & 3 & 60 & $(400,0,0)$ \\
USV2 & $(-362 \cdot 52,230 \cdot 95,0)$ & 2 & 25 & $(0,400,0)$ \\
USV 3 & $(-563 \cdot 83,114 \cdot 72,0)$ & 1 & -35 & $(-400,0,0)$ \\
USV 4 & $(-751 \cdot 75,-673 \cdot 62,0)$ & 4 & 20 & $(0,-400,0)$ \\
\hline
\end{tabular}

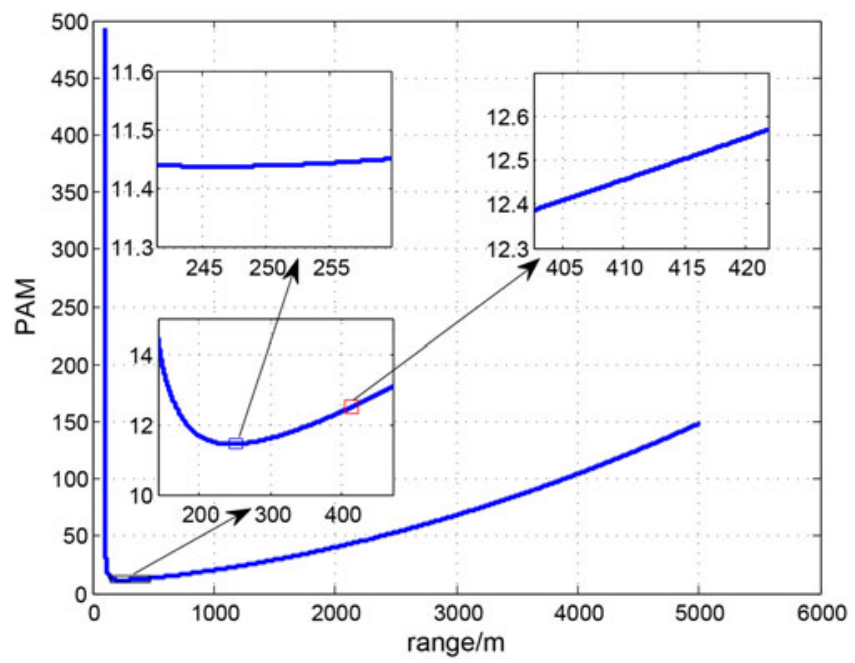

Figure 7. Relationship between the distance and the PAM.

positioning error $e_{k}$ can be acquired by averaging these positioning errors, i.e., $e_{k}=\frac{1}{100} \sum_{q=1}^{100} e_{q, k}$.

a) Optimal geometry: Two groups of USVs are used to position the target. One group consists of USV1, USV2, USV3 and USV4, and the other group consists of USV5, USV6, USV7 and USV8. Two groups are both in the optimal geometry originally. The parameters of the AUV and the USVs are shown in Table 2. The trajectories of the USVs are shown in Figure 8. The positions of the USVs and the AUV at $t=0 \mathrm{~s}$, $t=1000 \mathrm{~s}$ and $t=2000 \mathrm{~s}$ are denoted by ' $\mathbf{A}, ' \boldsymbol{\square}$ ' and ' $*$ ', respectively. Figure 9(a) shows the maximum and minimum positioning errors of 100 experiments in the optimal geometry, and Figure 9(b) shows the maximum and minimum positioning errors of 100 experiments in the general geometry. The mean positioning error is shown in Figure 10, and we can see that the group in the optimal geometry has a higher positioning accuracy.

b) Optimal distance: In this example, the optimal geometry has been reached. In Figure 11, three groups of USVs are used to position the target, and each group consists of four USVs. The distances in each group are $102.0 \mathrm{~m}, 246.2 \mathrm{~m}$ and $510.0 \mathrm{~m}$, respectively. Comparing these positioning errors, we find that the group at the optimal 
Table 2. The parameters of the USV and the AUV.

\begin{tabular}{lcccc}
\hline Vehicle & Initial Position $(\mathrm{m})$ & Velocity $(\mathrm{m} / \mathrm{s})$ & Yaw $\left({ }^{\circ}\right)$ & $\operatorname{Pitch}\left({ }^{\circ}\right)$ \\
\hline AUV & $(0,0,100)$ & $2 \cdot 003$ & 30 & 1 \\
USV1 & $(400,0,0)$ & 2 & 30 & \\
USV2 & $(0,400,0)$ & 2 & 30 & \\
USV 3 & $(-400,0,0)$ & 2 & 30 & \\
USV 4 & $(0,-400,0)$ & 2 & 30 & \\
USV5 & $(400,0,0)$ & 2 & 45 & \\
USV6 & $(0,400,0)$ & 3 & 10 & \\
USV7 & $(-400,0,0)$ & $1 \cdot 5$ & -10 & \\
USV8 & $(0,-400,0)$ & &
\end{tabular}

(a)

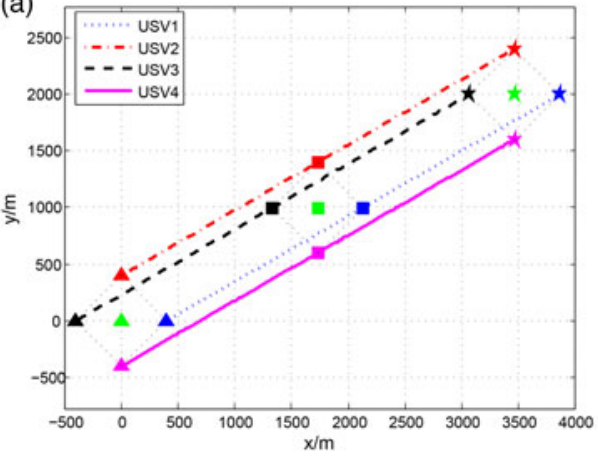

(b)

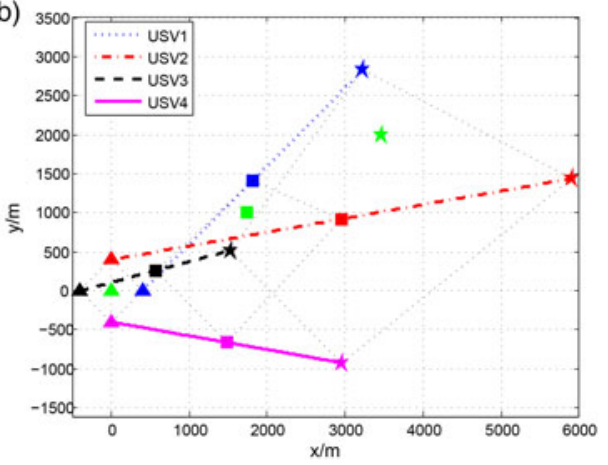

Figure 8. The trajectories of the USVs: (a) in optimal geometry, (b) in general geometry.

(a)

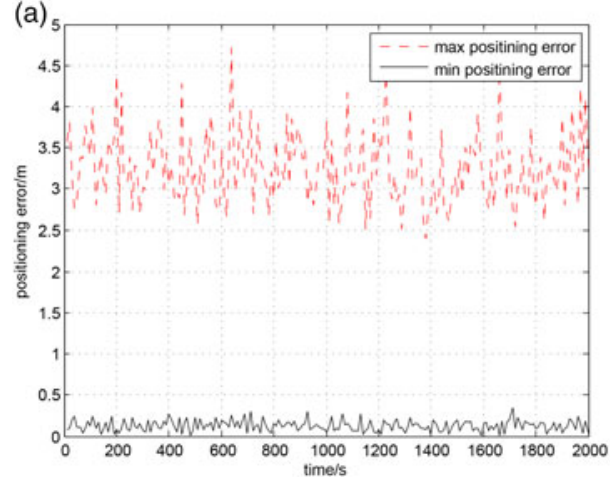

(b)

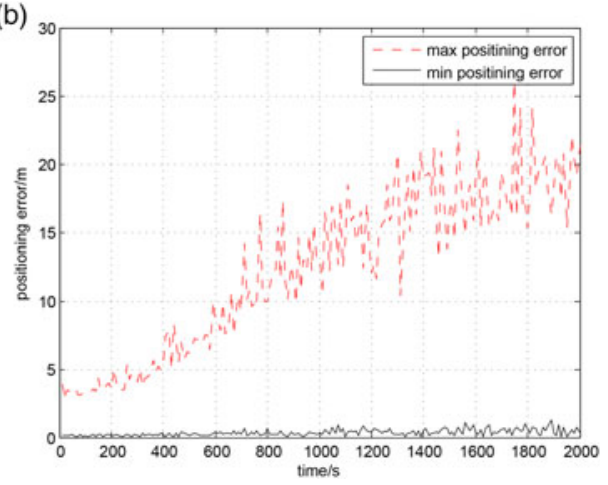

Figure 9. Maximum and minimum positioning errors of 100 experiments: (a) in optimal geometry, (b) in general geometry.

distance $r=246.2 \mathrm{~m}$ has the highest positioning accuracy. The positioning errors correspond to the results in Figure 7.

The simulation results indicate that the positioning accuracy depends directly on the PAM, and the optimal distance can apparently improve the positioning accuracy. 


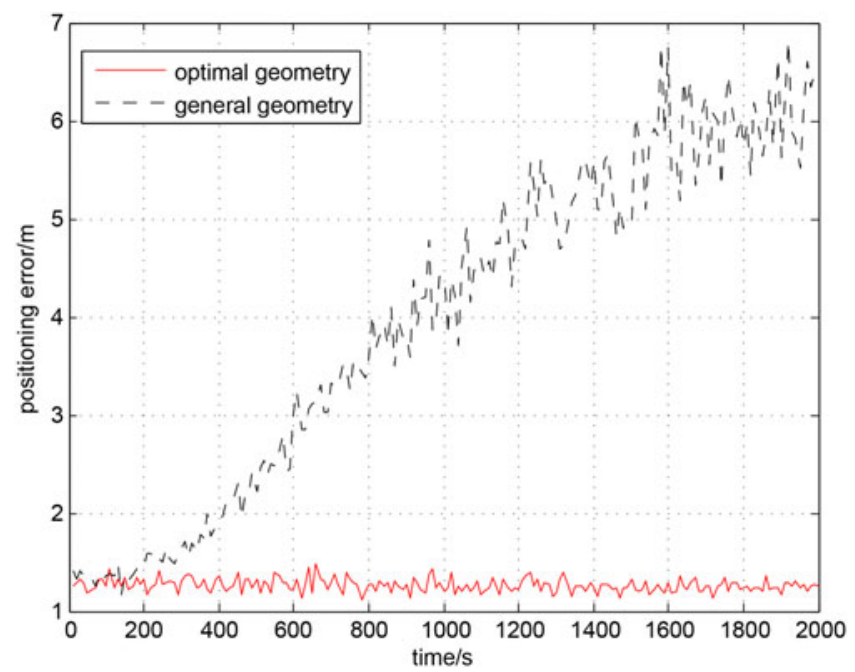

Figure 10. Comparison of the positioning error between optimal and general geometry.

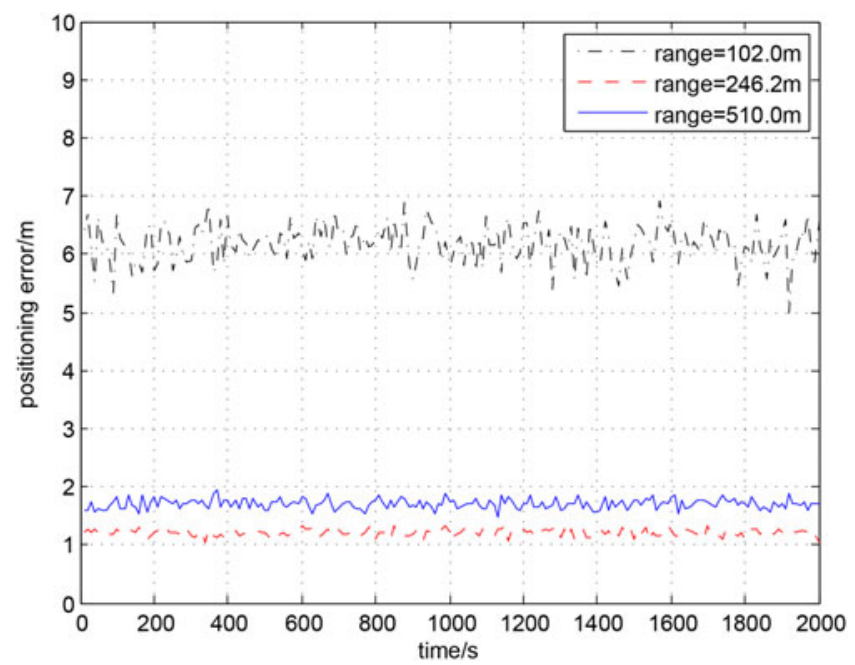

Figure 11. Relationship between the distance and the positioning error.

6. CONCLUSION. In this paper, we have investigated the problem of how to derive the optimal distance between the mobile buoy and the target to improve the positioning accuracy. The positioning model and error sources of MLBL have been developed. Considering the distance error and the position error of the mobile buoy, we propose the PAM to measure the positioning accuracy, based on which the optimal distance has been derived. We have shown that the optimal geometry is that the mobile buoys are on the vertices of the regular polygon centred at the target, and the 
optimal distance is related to the depth of the target and the parameter of the distance error. Simulation results have demonstrated the optimal distance and its effectiveness.

\section{ACKNOWLEDGMENTS}

This work was supported by the National Natural Science Foundation of China (NSFC) under grant 51209174, 61472325 and 61273333, the NSFC-RS joint research project under grants 51311130137 in China and IE121414 in UK, and the State Key Laboratory of Robotics and System (HIT) under grant SKLRS-2012-MS-04.

\section{REFERENCES}

Alcocer, A., Oliveira, P., and Pascoal, A. (2006). Underwater acoustic positioning systems based on buoys with GPS. Proceedings of the $8^{\text {th }}$ European Conference on Underwater Acoustics, Carvoerio, Portugal.

Alcocer, A., Oliveira, P., and Pascoal, A. (2007). Study and implementation of an EKF GIB-based underwater positioning system. Control Engineering Practice, 15(6), 689-701.

Batista, P., Silvestre, C., and Oliveira, P. (2014). Sensor-based Long Baseline Navigation: observability analysis and filter design. Asian Journal of Control, 16(3), 1-21.

Beaujean, P. P. J., Bon, A., and An, E. (2010). Motion-compensated acoustic positioning in very shallow waters using spread-spectrum signaling and a tetrahedral ultrashort baseline array. Marine Technology Society Journal, 44(5), 50-66.

Bertsekas, D. P. (1982). Constrained Optimization and Lagrange Multiplier Methods. Computer Science and Applied Mathematics, Academic Press, Inc.

Caiti, A., Garulli, A., Livide, F., and Prattichizzo, D. (2005). Localization of autonomous underwater vehicles by floating acoustic buoys: a set-membership approach. IEEE Journal of Oceanic Engineering, 30(1), $140-152$.

Erol-Kantarci, M., Mouftah, H. T. and Oktug, S. (2011). A survey of architectures and localization techniques for underwater acoustic sensor networks. Communications Surveys and Tutorials, 13(3), 487-502.

Fox, D., Burgard, W., Kruppa, H., and Thrun, S. (2000). A probabilistic approach to collaborative multirobot localization. Autonomous robots, 8(3), 325-344.

Howard, A., Matark, M. J., and Sukhatme, G. (2002). Localization for mobile robot teams using maximum likelihood estimation. Proceedings of the IEEE/RSJ International Conference on Intelligent Robots and Systems, EPFL, Switzerland.

Isik, M. T., and Akan, O. B. (2009). A three dimensional localization algorithm for underwater acoustic sensor networks. IEEE Transactions on Wireless Communications, 8(9), 4457-4463.

Jakuba, M. V., Roman, C. N., Singh, H., Murphy, C., Kunz, C., Willis, C., Sato, T. and Sohn, R. A. (2008). Long-baseline acoustic navigation for under-ice autonomous underwater vehicle operations. Journal of Field Robotics, 25(11-12), 861-879.

Jourdan, D. B., and Roy, N. (2008). Optimal sensor placement for agent localization. ACM Transactions on Sensor Networks, 4(3), 13.

Kaplan, E. D., and Hegarty, C. J. (2005). Understanding GPS: Principles and Applications. Artech house, Inc.

Kinsey, J. C., Eustice, R. M., and Whitcomb, L. L. (2006). A survey of underwater vehicle navigation: Recent advances and new challenges. Proceedings of the $7^{\text {th }}$ IFAC conference on manoeuvring and control of marine craft, Lisboa, Portugal.

Kussat, N. H., Chadwell, C. D., and Zimmerman, R. (2005). Absolute positioning of an autonomous underwater vehicle using GPS and acoustic measurements. IEEE Journal of Oceanic Engineering, 30(1), 153164.

Liu, B., Chen, H., Zhong, Z., and Poor, H. V. (2010). Asymmetrical round trip based synchronization-free localization in large-scale underwater sensor networks. IEEE Transactions on Wireless Communications, 9 (11), 3532-3542.

Lütkepohl, H. (1996). Handbook of Matrices, Wiley, Inc. 
MartíNez, S., and Bullo, F. (2006). Optimal sensor placement and motion coordination for target tracking. Automatica, 42(4), 661-668.

Moradi, M., Rezazadeh, J. and Ismail, A. S. (2012). A reverse localization scheme for underwater acoustic sensor networks. Sensors, 12(4), 4352-4380.

Moreno-Salinas, D., Pascoal, A. M., and Aranda, J. (2011). Optimal sensor placement for underwater positioning with uncertainty in the target location. Proceedings of the IEEE International Conference on Robotics and Automation, Shanghai, China.

Moreno-Salinas, D., Pascoal, A. M., and Aranda, J. (2012). Surface sensor networks for Underwater Vehicle positioning with bearings-only measurements. Proceedings of the IEEE/RSJ International Conference on Intelligent Robots and Systems, Vilamoura, Portugal.

Nickalls, R.W.D. (1993). A new approach to solving the cubic: Cardan's solution revealed. The Mathematical Gazette, 77, 354-359.

Olson, E., Leonard, J. J., and Teller, S. (2006). Robust range-only beacon localization. IEEE Journal of Oceanic Engineering, 31(4), 949-958.

Oshman, Y., and Davidson, P. (1999). Optimization of observer trajectories for bearings-only target localization. IEEE Transactions on Aerospace and Electronic Systems, 35(3), 892-902.

Philips, D. (2003). An evaluation of USBL and SBL acoustic systems and the optimisation of methods of calibration-part 2. The Hydrographic Journal, 109, 10-20.

Purvis, K. B., Astrom, K. J., and Khammash, M. (2008). Estimation and optimal configurations for localization using cooperative UAVs. IEEE Transactions on Control Systems Technology, 16(5), 947-958.

Savvides, A., Garber, W. L., Moses, R. L., and Srivastava, M. B. (2005). An analysis of error inducing parameters in multihop sensor node localization. IEEE Transactions on Mobile Computing, 4(6), 567-577.

Sukkarieh, S., Nebot, E. M. and Durrant-Whyte, H. F. (1999). A high integrity IMU/GPS navigation loop for autonomous land vehicle applications. IEEE Transactions on Robotics and Automation, 15(3), $572-578$.

Tan, H. P., Diamant, R., Seah, W. K., and Waldmeyer, M. (2011). A survey of techniques and challenges in underwater localization. Ocean Engineering, 38(14), 1663-1676.

Tan, L., Zhong-ying, Q., and Hong-yuan, N. (2009). A study of agricultural futures market simulation based on SBL model. Proceedings of the $1^{\text {th }}$ ICMSE on Management Science and Engineering, Zhuhai, China.

Teymorian, A. Y., Cheng, W., Ma, L., Cheng, X., Lu, X., and Lu, Z. (2009). 3D underwater sensor network localization. IEEE Transactions on Mobile Computing, 8(12), 1610-1621.

Vaganay, J., Leonard, J. J., Curcio, J. A., and Willcox, J. S. (2004). Experimental validation of the moving long base-line navigation concept. Proceedings of the IEEE/OES International Conference on Autonomous Underwater Vehicles, Nagoya, Japan.

Waite, A. D. (2002). Sonar for Practising Engineers. John Wiley and Sons, Inc.

Williams, R. (1979). The Geometrical Foundation of Natural Structure. Dover Publications, Inc.

Van Trees, H. L. (2004). Detection, Estimation, and Modulation Theory, Optimum Array Processing. John Wiley and Sons, Inc.

$\mathrm{Xu}$, Y., and Choi, J. (2011). Adaptive sampling for learning Gaussian processes using mobile sensor networks. Sensors, 11(3), 3051-3066.

Yun, X., Bachmann, E. R., McGhee, R. B., Whalen, R. H., Roberts, R. L., Knapp, R. G., Healey, A. J. and Zyda, M. J. (1999). Testing and evaluation of an integrated GPS/INS system for small AUV navigation. IEEE Journal of Oceanic Engineering, 24(3), 396-404. 